\title{
Swallowing Detection for Game Control: Using Skin-Like Electronics to Support People with Dysphagia
}

\author{
Benjamin Nicholls ${ }^{\dagger}$, Yongkuk Lee*, Woon Hong Yeo*, Chee Siang Ang ${ }^{\dagger}$, Christos Efstratiou ${ }^{\dagger}$ \\ † School of Engineering and Digital Arts, University of Kent, United Kingdom \\ Email:bpn2@kent.ac.uk ; c.s.ang@kent.ac.uk ; c.efstratiou@kent.ac.uk \\ * Department of Mechanical and Nuclear Engineering, School of Engineering \\ Virginia Commonwealth University, Richmond, USA \\ Email:ylee3@vcu.edu ; whyeo@vcu.edu
}

\begin{abstract}
In this work we explore the feasibility of developing a sensor-driven rehabilitation game for people suffering from dysphagia. This study explores the viability of skin-like electronics for unobtrusive, continuous recording of surface electromyograms (EMG) and use for driving game-based, user-controlled feedback. The experimental study includes the development end evaluation of a light-weight real-time swallow detection algorithm using skin-like sensors, and development of game-based HCI driven by skin-like electrodes. The results from a user evaluation are promising emphasising the ease of use of the overall system as a motivational tool for people with dysphagia.
\end{abstract}

\section{Introduction}

Dysphagia is a condition that affects the ability of a person to swallow. Managing this condition requires regular swallowing exercises that patients need to perform on a daily basis. As other cases of rehabilitation support have shown, wearable sensing technologies can play a significant role in monitoring the condition of a particular disease, and motivating patients to follow prescribed exercise regimes. However, when considering the case of dysphagia and odynophagia (painful swallowing) available wearable technologies either do not offer the right sensing modalities, or cannot be applied to the relevant parts of the body.

Novel sensing technologies that can easily be attached to any parts of the human body, can potentially detect the swallowing activities of patients suffering from dysphagia. Such technologies could in turn allow the development of biofeedback motivational tools as part of the daily rehabilitation routine of such patients. In this work we explore the feasibility of developing a sensor-driven training game for people suffering from dysphagia. In particular we use novel skin-like electromyography (EMG) sensors (Fig. 1) paired with a "swallow-drives" computer game to support the rehabilitation of such patients.

Wearable sensors as part of human-machine interaction system can allow the development of motivational tools to facilitate regular rehabilitation exercises. Accurate detection of the swallowing activities can be used as input to a computer game that would act as both a motivational tool, and as tracking instrument to record the performed swallowing exercises. In clinical settings, EMG sensing has been suggested as a means of supporting the screening of dysphagia and odynophagia [1], and for biofeedback therapy for dysphagia [2]. However, traditional approaches in detecting swallowing activity, involve the use of rigid body sensors applied temporarily to the face and neck using adhesive patches, and connected to physiological data collection devices. While existing clinical techniques are suitable for short-term assessment or therapy sessions, their bulky and obtrusive properties make them ill-suited to continuous, long term and mobile monitoring. In the context of rehabilitation support, there is a need for a light-weight, sensing solution that is easy to apply on the user's neck.

In this paper, we utilise a novel electronic system ('skin-like electronics') for recording electrophysiological signals (EMG) and driving interactive software. The skin-like electrodes are unobtrusive and comfortable to wear on the skin, while providing surface 


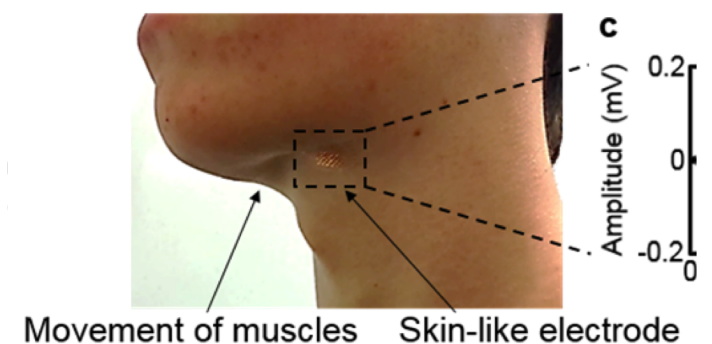

Figure 1. Skin-like EMG sensor.

conformal contact for high quality signal recording without the use of conductive gels. EMG signals from the masseter and submental muscles are used to detect deglutition (swallowing) and mastication (chewing). We use the deglutition (swallowing) behaviour, measured by EMG signals, to demonstrate game-based biofeedback. Real-time analysis of the EMG signals captured by the skin-like sensors, allow the detection of swallowing activities with minimal delay, sufficient to enable the interaction with an arcade-type computer game. The motivation of this work is to support swallowing exercises that need to be performed regularly by users, over relatively short session (approximately 15 mins). An arcade-type computer game that users can play with for short sessions using swallowing as input, was considered appropriate for this application.

The aim of this study is to demonstrate the feasibility of using unobtrusive skin-like electronics as a physiological sensing platform for game interactions. The main contributions of this work are:

- Analysis of the EMG signals captured through flexible skin sensors during deglutition (swallowing).

- Development of a light-weight signal processing system that can accurately detect swallowing activities through the EMG signal.

- Demonstration of the use of swallowing activities as input to game applications

\section{Related Work}

The use of physiological sensing has been suggested as a means of enhancing interaction with technology [3]. A practical application of this idea is the use of physiological signals to enhance gameplay. Nacke et al. [4] examined a range of physiological signals to best determine their use for game interaction. They concluded that voluntary and involuntary physiological signals such as EMG, body temperature, galvanic skin response (GSR), or heart rate can be used for direct game manipulation (in the case of voluntary signal
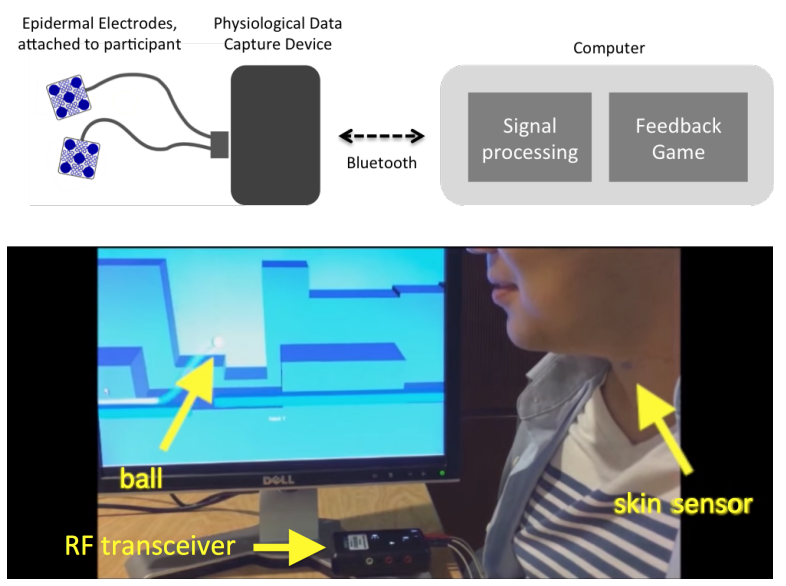

Figure 2. System architecture. The skin-like sensors transmit EMG signals to the computer. Swallowing detection is used to drive the arcade game.

responses) or to indirectly adapt the game environment (through sensing of involuntary reactions). Chanel et al. [5] similarly studied the use of EEG to monitor pressure, pleasure, arousal, and motivation to estimate emotional states of boredom, engagement and anxiety. This could be used to adapt difficulty in order to maintain the ideal state of engagement.

Conventional physiological measurement on the skin involves sensors being affixed to the skin using adhesive tape [6], which makes it unsuited to mobile or continuous usage. In recent years, researchers have focused on the development of novel bioelectronics that can be directly and conformably mounted on the skin [7]. Skin-like electronics (also known as "epidermal" electronics) are ultra-thin, ultra-light, and stretchable to minimize the thermal and mechanical loadings to the skin. The small form factor of the skin-like electronics have offered comfortable, intimate integration with the skin, without causing motion constraints. Our prior works demonstrated the use of such electronics for long-term ( $>2$ weeks) recording of EMG, ECG, and EEG signals [8], precise temperature mapping [9], thermal conductivity [10], hydration [11], and skinlike stimulation [12]. In this work, we explore the use of such sensors as inputs for gameplay interaction, in order to develop a rehabilitation support system for people suffering from dysphagia.

\section{Biofeedback System for Swallowing}

In order to support the swallowing exercises for patients with dysphagia we developed a system that allows users to interact with an arcade game by de- 


\section{EMG recording}

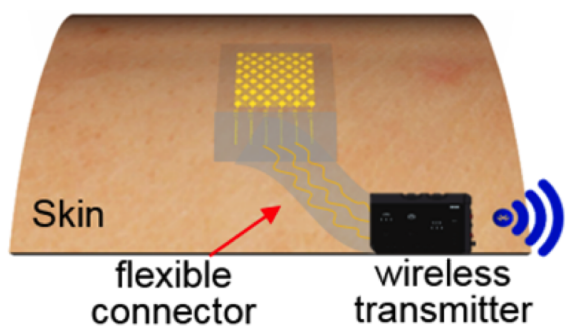

Figure 3. Skin mounted sensors with wireless communication unit.

tecting the muscle movements when they swallow ${ }^{1}$. The detection of swallowing required the capture of electromyography (EMG) signals and the processing of such signals to identify swallowing patterns (Fig. 2).

To measure surface EMG during the swallowing process, we fabricated a set of skin-like electrodes by following the microfabrication process from our prior works [8]. We used two types of soft elastomeric membranes (transparent silicone) to integrate the gold nanomembrane electrode (Fig. 3). Without the use of conductive gels and adhesives, ultrathin elastomeric membranes provided sufficient adhesion force to mount electrodes on the skin purely via van der Waals interactions. The elastomer-silky fabric material allowed multiple cycles of cleaning and reuse to measure EMG on the skin, while transparent silicone ( $5 \mu \mathrm{m}$ in thickness) offered long-term lamination on the skin. In combination this provided unobtrusive, comfortable, soft lamination of electrodes on the skin. A flexible, ultrathin carbon cable connected the electrode to the wireless recording device. The recorded analogue EMG signals were converted and amplified in the device and wirelessly transmitted to the receiver via Bluetooth for continuous data recording.

\subsection{Swallow detection}

Six participants (three females and three males) between 21 and 40 years of age in good health (with no reported medical conditions) were recruited to take part in this research by following the approved protocol at Virginia Commonwealth University (approved number: HM20001454). Each participant underwent EMG data collection using skin-like sensors during a number of exercises intended to acquire information about

1. You can watch a video of the system in action here: https://youtu.be/PIBkpdqbGjA deglutition and mastication (a total of 432 swallows were collected). EMG signals were recorded each session at a sample rate of $1 \mathrm{kHz}$. During the sessions, the participants were asked to perform a number of swallowing exercises, giving an indication of muscular activity during swallowing behaviours. The types of swallows that they asked to perform included:

- Dry swallow: 15 trials of voluntary dry swallows (saliva swallowing).

- Liquid swallow: 15 trials of voluntary swallowing of a small mouthful of water.

- Extended swallow (Mendelsohn Maneuver): An exercise performed to help improve swallowing, raising the larynx and opening the oesophagus [Burkhead et al. 2007]. The participant performed a dry swallow and at the peak of the swallow the participants attempted to hold the swallow for 2 seconds, before releasing. This exercise was repeated 5 times.

During each data acquisition session, synchronised video footage was recorded. The recorded video clips provided manual extraction of the time and duration of each swallow event and also allowed the identification of corresponding EMG activity and the ground truth of these events.

After completing data collection, the data was processed in order to analyse the swallowing EMG signals. In order to eliminate high frequency noise and movement artefacts, the data was filtered using a band pass filter to extract the frequencies of interest from the signals. A windowed root mean square was then applied across the data providing an informative interpretation of the filtered muscle activity. The signals were further smoothed for root-mean-square (RMS) signals using a windowed average in a similar manner (Fig. 4). The data classification algorithm included magnitude and duration thresholds to detect segments of active signal that exceeded the signal baseline for a significant duration. Due to the variability of the recorded signals between participants, these threshold values were calibrated individually for each participant.

The primary magnitude threshold was used to check for instances of the signal magnitude exceeding the threshold, which could then be associated with swallowing periods. A duration threshold was used to ensure that the signal remained above the primary threshold for a given duration (as set during calibration). Once the signal was considered to be demonstrating muscle activity, the same technique was used to determine the end of the signal segment, ensuring that the activity was only considered complete when the magnitude dropped back below the primary magnitude 

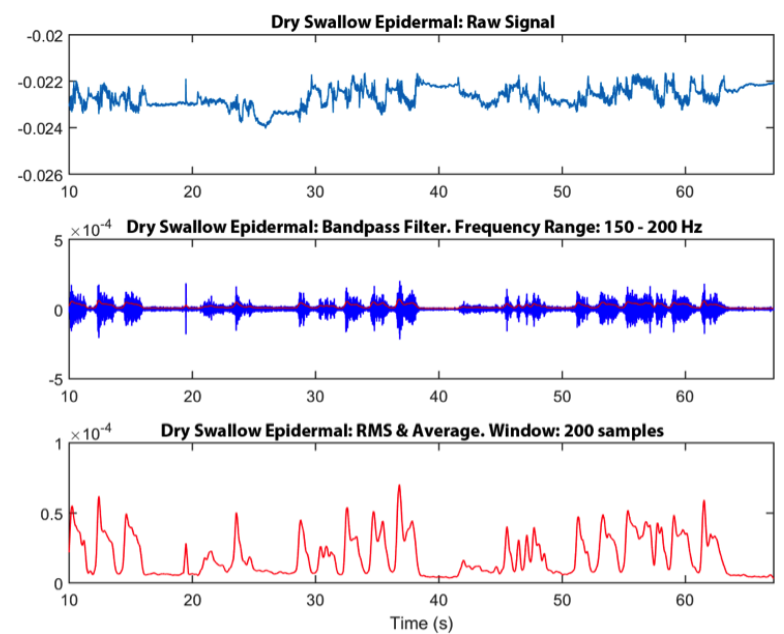

Figure 4. Representative EMG data (submental muscles) collected using a set of skin-like sensors during swallowing with participant 1: raw EMG signals (top), filtered signals (middle), and smoothed RMS signals (bottom).

threshold and remained there for a number of samples indicated by the duration threshold.

The start and end points of the target segment were then more precisely identified by locating the peaks of the inverse of the signal, using the first trough before the estimated signal start as the true start of the signal, and the first trough after the estimated end as the true activity segment end. Together these features ensured that the activity segments identified would consist of a signal above a certain level for at least a given duration and display a significant peak.

We evaluated the thresholding technique for detecting swallowing by comparing the results with the manually annotated video feed. As shown in Table 2 although the study is relatively limited in scale, the results are very promising. Employing a simple thresholding technique to identify swallows, the algorithm maintains an average detection error of $1.3 \%$ for the dry swallow and $3.3 \%$ for the liquid swallow. The extended swallow appeared to be more challenging to detect with this technique, with an average error of $13.8 \%$.

\section{Biofeedback Game}

The processing technique used previously was adapted to analyze the EMG signals and identify active periods in real time using a custom-developed data processing application. It was then possible to investigate the use of this system as a means for driving user-
Table 1. Results of the swallow detection algorithm

\begin{tabular}{l|l|l|l}
\hline (per participant) & $\begin{array}{l}\text { Ground } \\
\text { Truth }\end{array}$ & $\begin{array}{l}\text { Mean } \\
\text { Detected } \pm \text { SD }\end{array}$ & Error \pm SD \\
\hline \hline Dry Swallow & 15 & $14.80 \pm 1.92$ & $-0.20 \pm 1.92$ \\
Liquid Swallow & 15 & $15.50 \pm 0.55$ & $0.50 \pm 0.55$ \\
Extended Swallow & 6 & $6.83 \pm 1.83$ & $0.83 \pm 0.98$ \\
\hline
\end{tabular}

friendly feedback software (feedback-based game). Finally, we carried out a user evaluation to determine the user experience and opinion of the control method.

The full system is described in Fig. 2. Skin sensors are used to capture EMG signals, that are then transmitted to a computer over Bluetooth. A swallow detection algorithm is then employed to detect swallow activities in real-time. Processing was performed every 0.125 seconds (125 samples) upon an overlapping signal segment 0.25 seconds ( 250 samples) long. Such events are used to drive an arcade computer game.

The processing application detected muscle activity in a binary manner. After the successful detection of the start of a period of muscle activity, the application would respond with a positive indicator for muscle activity until such time as an end of this period of activity was detected. This binary information could then be passed to the feedback-based game in order to drive its responses. Because of the individual variability in swallow magnitude and duration, as indicated by the first stage of the study, the threshold values were made fully adjustable using the software application, to allow the user (in this case the supervising researcher) to calibrate the values according to observed signal magnitudes and trends.

Biofeedback has been used in rehabilitation for a number of conditions, including the swallowing disorders our study focuses upon. Moreover, serious games have been demonstrated as useful in the treatment and rehabilitation of conditions [13]. As such a simple game was considered as a viable format for the evaluation of feedback using this system.

The simple game was designed to convey to the user when their muscles were considered active (a swallow was detected) in the EMG signal, and the duration they maintained this activity (Fig. 5). The game includes a ball that travels at a steady rate along a series of platforms. A value read from the input stream positively indicating muscle activity results in the ball jumping. While the muscle activity is maintained (e.g. Mendelsohn Maneuver), the duration is shown in the game. While the value remains constantly active, the ball does not cease 'jumping'. However, 

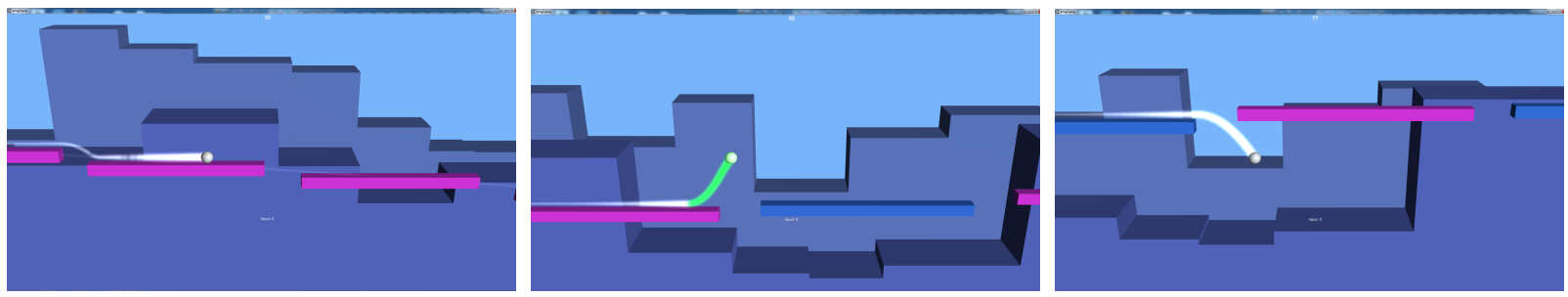

Figure 5. Swallow driven game: A ball is traveling across the platofrm. A swallow can make the ball jump between platforms. Coloured trail indicates signal activity. Green tail during swallowing.

as soon as the input (swallowing behaviour) is no longer found, the 'jump' is released and the ball falls onto a platform. Details of the game control via a skin-like electrode can be found in this video clip: https://youtu.be/PIBkpdqbGjA.

\section{Evaluation}

A user evaluation was conducted to investigate the viability of the use of skin-like sensing as a technique for driving user-friendly feedback. In particular, we hoped to examine the proposed solution's capabilities in swallowing disorder

The same six participants were asked to take part in the evaluation. During the study the participants attempted to control the game by dry swallowing. They were given an unlimited time to attempt to guide the game ball through the game, traversing gaps by swallowing to trigger a 'jump'. They were instructed to continue for as long as they could manage, or until they could no longer comfortably dry swallow - at which point they should cease attempting to play and allow the game to end. Once the ball had fallen into a gap the game ended and the participants were provided with a drink of water and given up to two minutes to rest. At this point the participants were challenged to beat their previous score, the game restarted and the process repeated. The participants were given 5 attempts at the game. During each session, video footage of the participants and the game-play feedback was recorded, allowing information to be extracted regarding the game response and participant control.

At the end of evaluation sessions, each participant took part in an evaluation interview, to offer insight into the acceptability and appropriateness of the skinlike sensors for user interaction and monitoring. The questions were split into a number of topics, including sensor comfort and wearing the sensor itself, perceived potential comfort of using the sensor in public situations ('home/office') and any concerns, and the potential comfort levels which might be involved
Table 2. Game response: success and failure rate, and mean false positives.

\begin{tabular}{c|c|c|c|c|c|c}
\hline$\#$ & 1 & 2 & 3 & 4 & 5 & 6 \\
\hline \hline Hits \% & 100.0 & 78.6 & 90.0 & 100.0 & 96.3 & 92.3 \\
Miss \% & 0.0 & 21.4 & 10.0 & 0.0 & 3.7 & 7.7 \\
FP & 3.4 & 0.4 & 0.8 & 0.0 & 1.2 & 2.0 \\
\hline
\end{tabular}

in prolonged use of the sensor. The final question concerned the control of the game-based feedback and its ease of use. Each topic was answered on a 7-point Likert scale.

Data were manually extracted from the evaluation video trials to assess the performance of the gamebased feedback and participant success rate. This included an estimated tally of successful responses (swallow followed by a successful game response), failed responses (swallow without any game response), and false positives (game response without any observable preceding swallow, or following a non-deglutition behavior). Response time (delay between swallow behavior and game response) was also recorded based on video footage, along with the total duration participants managed to maintain gameplay in each attempt.

The results demonstrated a high percentage of successful responses per gameplay as summarised in Table 4. The rate of failed game responses was not indicative of the performance of the system. Because of the nature of the feedback game, a failure to respond to a single swallow was likely to result in the participant 'losing' and the game resetting, leading to a low failed response rate. False positives on the other hand occur more frequently, with a mean of 1.3 per game. As shown in the results, distribution of false positives was mostly biased towards participant 1 , participant 5 , and participant 6; the other participants in comparison each demonstrated a mean number of false positives less than 1. This can be an indication that the the use of a simple thresholding technique may not be very robust to minor fluctuations on the EMG signal. 
Table 3. Evaluation ratings of interview questions (7-point scale)

\begin{tabular}{c|l|l|l|l}
\hline & $\begin{array}{l}\text { Sensor } \\
\text { Comfort }\end{array}$ & $\begin{array}{l}\text { Different } \\
\text { Environ. }\end{array}$ & $\begin{array}{l}\text { Long-Term } \\
\text { Use }\end{array}$ & $\begin{array}{l}\text { Game } \\
\text { Feedback }\end{array}$ \\
\hline \hline Mean & 6 & 5.83 & 4.5 & 5.16 \\
SD & 0.81 & 0.89 & 1.38 & 1.06 \\
\hline
\end{tabular}

In the interview results, the participants were generally very positive about the comfort of the sensor with an average mark of 6 out of 7 . On the other hand, four comments were made by participants stating that they did not feel comfortable wearing them, suggesting that they were still 'aware' of the sensors, that the sensors felt like a 'band-aid'. The sensors were praised for their ease of application and use, however there was concern regarding restricted motion caused by the wires and the wireless device used with the sensor. One comment highlighted the requirement to shave as a significant inconvenience. Two participants raised concerns about using the sensors with the adhesive patches on exposed areas of the body such as the face and one participant stated that it would be socially awkward.

For the game-based feedback question, separate areas of focus were evident in the responses, including the game-based feedback 'fun', the 'ease of swallowing' and the 'responsiveness' of the game. Three participants reported that using the game was âĂŸfunâĂŹ and two indicated that it made repeated swallowing easier ('Ease of swallowing'), or improved their focus upon swallowing. However, three participants also highlighted the difficulty involved when required to swallow repeatedly.

Finally, three participants made comments praising the mobility of the system, and all but one participant stated that they thought that the sensors and wireless communication might allow for continuous and interesting monitoring.

\section{Conclusions}

In this study, we demonstrated the potential of the skin-like electronics for unobtrusive lamination on the skin, high-fidelity EMG recording of swallowing behaviour, and the application for a game-control system. In our future work we intent to further develop the swallow detection algorithm, to improve its accuracy, while expanding the functionality of the game to respond to different types of swallowing.

\section{References}

[1] M. Vaiman and E. Eviatar, "Surface electromyography as a screening method for evaluation of dysphagia and odynophagia," Head \& face medicine, vol. 5, no. 1, p. 1, 2009.

[2] M. A. Crary, G. D. Carnaby, M. E. Groher, and E. Helseth, "Functional benefits of dysphagia therapy using adjunctive semg biofeedback," Dysphagia, vol. 19, no. 3, pp. 160-164, 2004.

[3] M. Pantic and L. J. Rothkrantz, "Toward an affectsensitive multimodal human-computer interaction," Proceedings of the IEEE, vol. 91, no. 9, pp. 1370-1390, 2003.

[4] L. E. Nacke, M. Kalyn, C. Lough, and R. L. Mandryk, "Biofeedback game design: using direct and indirect physiological control to enhance game interaction," in Proceedings of the SIGCHI conference on human factors in computing systems. ACM, 2011.

[5] G. Chanel, C. Rebetez, M. Bétrancourt, and T. Pun, "Emotion assessment from physiological signals for adaptation of game difficulty," IEEE Transactions on Systems, Man, and Cybernetics-Part A: Systems and Humans, vol. 41, no. 6, pp. 1052-1063, 2011.

[6] A. Searle and L. Kirkup, "A direct comparison of wet, dry and insulating bioelectric recording electrodes," Physiological measurement, vol. 21, no. 2, 2000.

[7] D.-H. Kim, N. Lu, R. Ma, Y.-S. Kim, R.-H. Kim, S. Wang, J. Wu, S. M. Won, H. Tao, A. Islam et al., "Epidermal electronics," science, vol. 333, no. 6044, pp. 838-843, 2011.

[8] J. J. Norton, D. S. Lee, J. W. Lee, W. Lee, O. Kwon, P. Won, S.-Y. Jung, H. Cheng, J.-W. Jeong, A. Akce et al., "Soft, curved electrode systems capable of integration on the auricle as a persistent brain-computer interface," Proceedings of the National Academy of Sciences, vol. 112, no. 13, pp. 3920-3925, 2015.

[9] R. C. Webb, A. P. Bonifas, A. Behnaz, Y. Zhang, K. J. Yu, H. Cheng, M. Shi, Z. Bian, Z. Liu, Y.-S. Kim et al., "Ultrathin conformal devices for precise and continuous thermal characterization of human skin," Nature materials, vol. 12, no. 10, pp. 938-944, 2013.

[10] Y. Hattori, L. Falgout, W. Lee, S.-Y. Jung, E. Poon, J. W. Lee, I. Na, A. Geisler, D. Sadhwani, Y. Zhang et al., "Multifunctional skin-like electronics for quantitative, clinical monitoring of cutaneous wound healing," Advanced healthcare materials, vol. 3, no. 10, pp. 1597-1607, 2014.

[11] X. Huang, W.-H. Yeo, Y. Liu, and J. A. Rogers, "Epidermal differential impedance sensor for conformal skin hydration monitoring," Biointerphases, vol. 7, no. 1, p. 52, 2012.

[12] B. Xu, A. Akhtar, Y. Liu, H. Chen, W.-H. Yeo, S. I. Park, B. Boyce, H. Kim, J. Yu, H.-Y. Lai et al., "An epidermal stimulation and sensing platform for sensorimotor prosthetic control, management of lower back exertion, and electrical muscle activation," Advanced Materials, 2015.

[13] J. W. Burke, M. McNeill, D. K. Charles, P. J. Morrow, J. H. Crosbie, and S. M. McDonough, "Optimising engagement for stroke rehabilitation using serious games," The Visual Computer, vol. 25, no. 12, pp. 1085-1099, 2009. 